\title{
Partitioning the symptoms of hypoglycaemia using multi-sample confirmatory factor analysis
}

\author{
I.J.Deary ${ }^{1}$, D.A.Hepburn ${ }^{2}$, K.M.MacLeod ${ }^{2}$, B.M.Frier ${ }^{2}$ \\ ${ }^{1}$ Department of Psychology, University of Edinburgh, Scotland, UK \\ ${ }^{2}$ Department of Diabetes, Royal Infirmary of Edinburgh, Scotland, UK
}

\begin{abstract}
Summary. The allocation of hypoglycaemic symptoms to autonomic or neuroglycopenic groups tends to occur on an a priori basis. In view of the practical need for clear symptom markers of hypoglycaemia more scientific approaches must be pursued. Substantial evidence is presented from two large scale studies we performed which support a three factor model of hypoglycaemic symptomatology, based on the statistical associations discovered among symptoms reported by diabetic patients. Study 1 involved 295 insulin-treated outpatients and found that 11 key hypoglycaemic symptoms segregated into three clear factors: autonomic (sweating, palpitation, shaking and hunger) neuroglycopenic (confusion, drowsiness, odd behaviour, speech difficulty and incoordination), and malaise (nausea and headache). The three factors were validated on a separate group of 303 insulin-treated
\end{abstract}

diabetic out-patients. Confirmatory factor analyses showed that the three factor model was the optimal model for explaining symptom covariance in each group. A multi-sample confirmatory factor analysis tested the rigorous assumptions that the relative loadings of symptoms on factors across groups were equal, and that the residual variance for each symptom was identical across groups. These assumptions were successful, indicating that the three factor model was replicated in detail across these two large samples. It is suggested that the results indicate valid groupings of symptoms that may be used in future research and in clinical practice.

Key words: Hypoglycaemia, neuroglycopenia, autonomic, factor analysis, insulin.
From the introduction of insulin for the treatment of diabetes mellitus the pattern of symptoms and signs which develops when blood glucose declines below normal was considered to be so characteristic that it was referred to as an "hypoglycaemic reaction" [1]. The symptoms of hypoglycaemia have usually been subdivided into those resulting from activation of the autonomic nervous system (autonomic or neurogenic symptoms) and those which develop from the direct effect of glucose deprivation on higher mental function (neuroglycopenic symptoms). The frequency with which diabetic patients report individual symptoms of hypoglycaemia has been assessed in retrospective studies [2-8] and in prospective studies $[9,10]$ of insulin-treated diabetic patients. Individual symptomatic responses vary between human subjects, and symptom profiles are also idiosyncratic in diabetic patients [11-13]; though within a subject symptoms appear to be characteristic and consistent over a short period of time.

The allocation of individual symptoms to autonomic and neuroglycopenic groups has been partially validated by experimental studies [14-17], but the hypothetical classification of other symptoms has been based on theoretical deduction. The classification of hypoglycaemic symptoms becomes important when accurate systems of symptom scoring are needed. In the past some studies of hypoglycaemia in humans $[18,19]$ have been criticised $[20$, 21] on the basis of imprecise classification of symptoms.

Using factor analysis Hepburn et al. [22] found a clear separation of neuroglycopenic and autonomic groups of symptoms in subjects undergoing insulin-induced hypoglycaemia in a laboratory setting. From a retrospective study of 295 insulin-treated diabetic patients there was evidence that there might be five separate groups of hypoglycaemic symptoms, viz. neuroglycopenic, autonomic, malaise, motor control and sensory dysfunction [8]. However, a three factor solution might offer a better division of the symptoms of hypoglycaemia. A third symptom factor, similar to the malaise factor described by Hepburn et al. [8], just failed to meet the criterion for acceptance as a factor in an earlier study by the same group [22]. In this latter study the number of subjects was such that the factor solution was very conservative, and likely to underestimate the number of factors. In the second study by Hepburn et al. [8] the much larger number of subjects facilitated the extraction of a larger number of factors. Moreover, both of these studies tended to include symptoms that were 
Table 1. Clinical characteristics of patients in Study 1 and Study 2

\begin{tabular}{lll}
\hline & Study 1 & Study 2 \\
\hline Subjects recruited $(n)$ & 302 & 305 \\
Age (years) & $44(16: 14-79)$ & $40(16: 13-76)$ \\
Duration of diabetes (years) & $16(11: 1-62)$ & $16(11: 1-49)$ \\
Duration of insulin therapy (years) & $15(11: 1-62)$ & $15(11: 1-49)$ \\
HbA $_{1}(\%)$ & $10.8(1.8: 6.0-16.4)$ & $10.1(1.8: 6.2-16.2)$ \\
\hline
\end{tabular}

Values are given as mean (SD: range)

likely to be rated similarly, such as incoordination and difficulty with walking, which can result in the extraction of specific mini-factors, especially in large subject samples.

The present study aims to provide a more definitive partitioning of the symptoms of hypoglycaemia into their underlying factors by using structural equation modelling [23]. A three factor model of hypoglycaemia was proposed to underlie 11 key symptoms of hypoglycaemia. Methodological improvements over previous studies included: (a) cross validation of the model using exploratory factor analysis in two large, independent groups of patients; (b) testing the model for goodness-of-fit in the two groups using confirmatory factor analysis; and (c) testing for similarity of the covariance structure of the symptom correlations between the two groups using multi-sample confirmatory factor analysis.

\section{Patients and methods}

Study 1. The diabetic patients included in the first study and the method of their selection have been described previously in detail [24]. Random number tables were used to select 302 attendees at daily diabetic clinics. All 302 insulin-treated patients agreed to participate and their clinical characteristics are given in Table 1. Seven of the 302 patients denied ever having experienced an episode of hypoglycaemia and were, therefore, excluded from further consideration. The remaining 295 patients had experienced hypoglycaemia and were included in the subsequent analyses; 34 of these subjects were insulin-treated Type 2 diabetic patients.

Study 2. A second study was conducted using insulin-treated diabetic patients who were recruited consecutively as they reported at the daily diabetic outpatient clinics at the Royal Infirmary of Edinburgh. This study was conducted 2 years after the first in order to validate the model of symptom relationships derived from Study 1 in a new sample. Three hundred and five patients who had received insulin treatment for more than 1 year were identified and all agreed to participate. None of the patients in Study 2 had taken part in Study 1. The clinical characteristics of these patients are given in Table 1. Of the 305 patients, 2 reported never having experienced hypoglycaemia while receiving insulin treatment; 22 of these subjects were insulin-treated Type 2 diabetic patients.

\section{Hypoglycaemia questionnaires}

Study 1. Patients were interviewed using a structured questionnaire as described previously [24]. The patients were asked what symptoms they usually experienced during episodes which they considered to be hypoglycaemic. In a retrospective study such as this it was not possible to verify hypoglycaemic episodes with biochemical evidence.

Study 2. Patients were asked to complete a proforma which listed 21 symptoms which had been included in the first study. The list of symptoms for this study changed the previous order of symptoms in order to avoid response sets. The patients were informed that the survey was to be used to provide evidence concerning their hypogly- caemic reactions. They were instructed to circle those symptoms which they recognised as being present during their own hypoglycaemic reactions.

\section{Statistical analysis}

On the basis of previous research $[8,22] 11$ key symptoms were identified as markers of three factors: sweating, palpitation, shaking and hunger were included as 'autonomic' symptoms; confusion, drowsiness, odd behaviour, speech difficulty and incoordination were chosen as neuroglycopenic symptoms; and nausea and headache were used as indicators of general malaise. These were chosen because they: (a) loaded moderately or highly on the factor for which they were markers, (b) had low loadings on other factors, (c) did not appear to be alternative wordings of the same underlying symptom, and (d) were endorsed by a sufficient proportion of patients to make them useful. Statistical analyses of the responses to these 11 symptoms took place in two stages, by exploratory and then confirmatory factor analyses.

Exploratory factor analysis. The responses to the 11 key symptoms given by the 295 subjects studied by Hepburn et al. [8] were analysed using principal components analysis followed by varimax rotation on the Statview program run on an Apple Macintosh computer. The criterion of Eigenvalues greater than one was used to extract factors (the Eigenvalue of a factor is indicative of how much variance it accounts for). The same analysis was conducted on responses to the same 11 symptoms given by 303 new subjects in order to test for cross-sample validation of the factor structure. Similarity of rotated factors between the two groups was assessed by inspection and by calculating the coefficients of congruence of the relevant factors.

Confirmatory factor analysis. More rigorous tests of the three factor model of hypoglycaemic symptoms were undertaken using structural equation modelling procedures. These analyses were run on an IBM PS/2 computer using the EQS structural equations package [23]. Whereas traditional exploratory factor analytic procedures allow the discovery of the underlying structure of a correlation matrix, they are essentially descriptive techniques where the formation of explicit models is driven by the data. Confirmatory factor analysis allows an explicit model to be articulated in advance of the data being examined, and this model may then be tested for its goodnessof-fit to the data using a variety of testing procedures. In addition, alternative models may be tested in competition with the stipulated model, and decisions made concerning the best model. Therefore, confirmatory factor analysis represents a major advance which allows hypothesis testing to be conducted in a field that has until recently been merely descriptive.

For the present report a structural equation model explicitly describing the proposed three factor model of hypoglycaemia was constructed and tested for its goodness-of-fit to the data in symptom covariance matrices comprising the results of Studies 1 and 2 . The method of Generalised Least Squares was used to test the hypothesis that data in Studies 1 and 2 fitted a three factor model of neuroglycopenic, autonomic and malaise factors. Chi-square tests of goodness-of-fit and Bentler-Bonett fit indices were used to test the adequacy of the models. Competing models were considered using the Wald and Lagrange Multiplier tests. The Wald test indicates whether parameters included in the model may be dropped without a significant loss of model fit; it explores the data for a more parsimonious model that has a better or equally good fit to the data. The Lagrange Multiplier test indicates any fixed parameters in the model that might be freed in order to give a better model fit. Because all fixed parameters in this investigation were those which were fixed at zero, the Lagrange Multiplier test in effect was used to indicate significant relationships which should be included to attain better model fitting but which were not included in the hypothesised three factor model.

Multi-sample confirmatory factor analysis was then applied. This is a more rigorous cross-sample validation of model similarity. Beyond establishing that the same three factor model was the best description of both data sets, this procedure was used to test whether the symptom loadings within the respective factors and the residual 
Table 2. Plan of statistical analyses of the data from Study $1(n=295)$ and Study $2(n=303)$. Hypothesis: three factors underlie 11 key symptoms of hypoglycaemia. The data to be analysed are contained in the matrix of symptom correlations for the exploratory factor analyses and the symptom covariance matrix for the confirmatory factor analyses

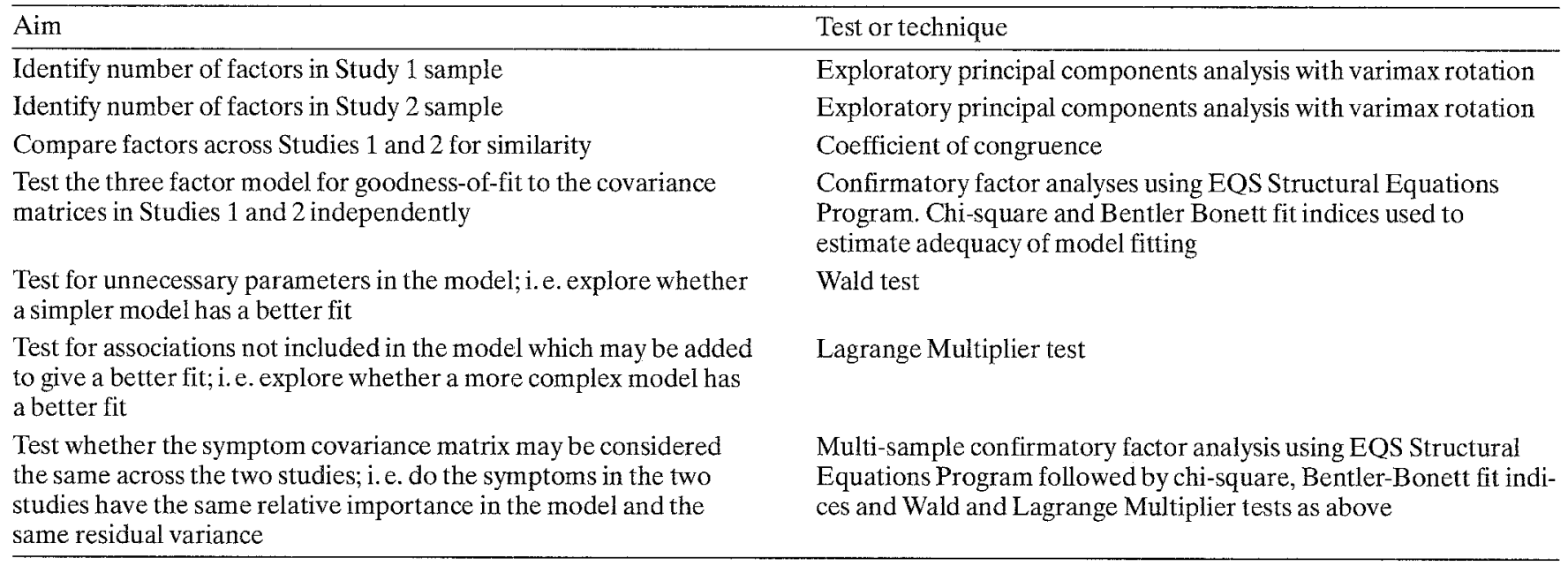

Table 3. Principal components analyses followed by varimax rotation of data collected on 11 key symptoms in Study $1(n=295)$ and in Study 2 $(n=303)$

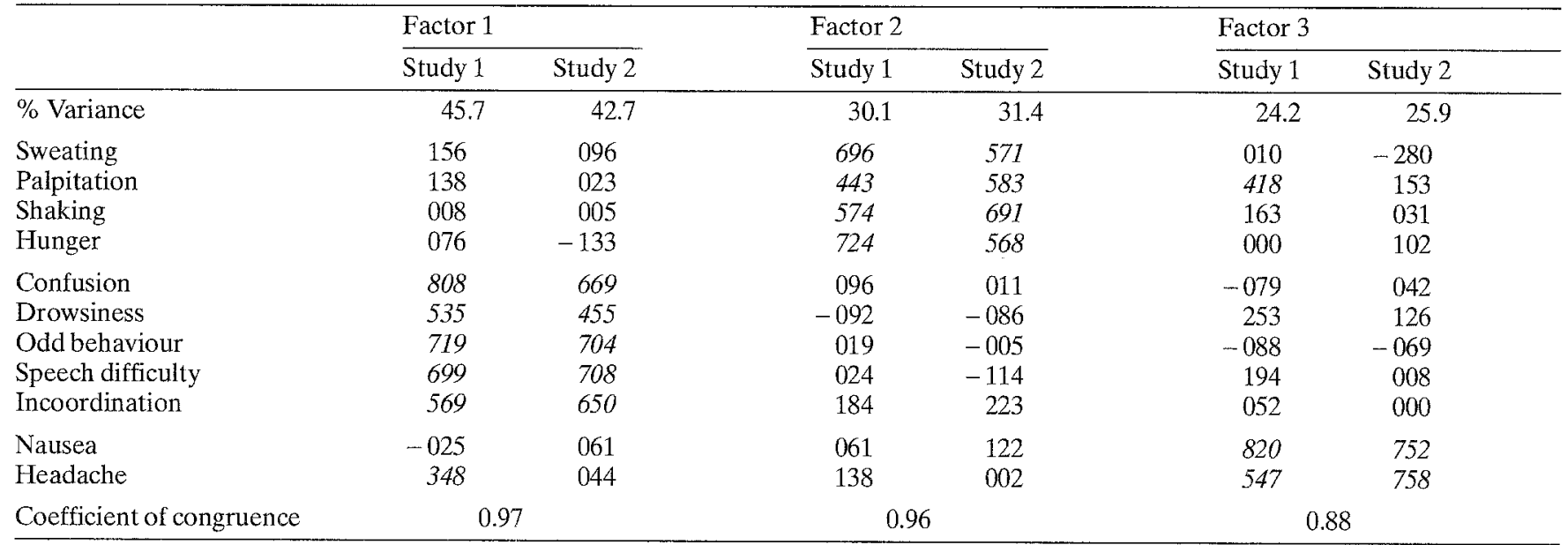

Decimal points are omitted from the factor loadings. Only rotated factors are shown. Loadings greater than 0.3 are shown in italic type

symptom variance in the two studies were identical. The method of Generalised Least Squares was used to test the adequacy of the model constructed to formulate this hypothesis.

Because some of the techniques used in this study are likely to be unfamiliar to many readers, and to make explicit the logical train of analyses that were conducted in order to achieve rigorous validation of the three factor model, Table 2 contains the stages of the statistical analysis in this investigation, and indicates the nature and purpose of the tests used at each stage.

\section{Results}

\section{Exploratory factor analysis}

Study 1. Correlations among the 11 key hypoglycaemic symptoms rated by the 295 subjects in Study 1 [8] were submitted to principal components analysis followed by varimax rotation. Factors were extracted using the criterion of Eigenvalues greater than one. Given the large number of subjects, loadings greater than about 0.2 are significant at the $1 \%$ level (Table C.3 in [25]) but, to emphasise the larger, more important loadings, only those above 0.3 are shown in italic type in Table 3 . Three factors had Eigenvalues greater than one, and accounted for $48.6 \%$ of the total variance. After rotation three orthogonal factors were obtained, as shown in Table 3. The first factor, accounting for $45.7 \%$ of the shared variance, had high loadings for confusion, drowsiness, odd behaviour, difficulty speaking and incoordination, and a lower but significant loading for headache. This was termed a 'neuroglycopenic' factor.

The second rotated factor accounted for $30.1 \%$ of the common variance and had high loadings for sweating, shaking and hunger, and a moderate loading for palpitation (Table 3). This was termed an 'autonomic' factor. The third factor accounted for $24.2 \%$ of the common variance and had high loadings for nausea and headache, and a moderate loading for palpitation (Table 3 ). This was entitled a 'malaise' factor.

The importance of factors lies in the absence of unexpected loadings in addition to the presence of expected loadings. On this criterion the two most important factors were well separated. Table 3 shows that in Study 1 the neuroglycopenic factor was free from any significant load- 
ings on autonomic-type symptoms. The autonomic factor had no significant loadings for neuroglycopenic symptoms. The malaise factor was the only one with slight lack of clear separation. Palpitation, an autonomic symptom, and drowsiness, a neuroglycopenic symptom, had significant loadings on this factor, though these were lower than those of the two main symptoms, nausea and headache. Headache had a significant loading on the neuroglycopenic factor, though this was markedly lower than the loadings of the five main symptoms.

Study 2. The same 11 symptoms were rated for their presence in hypoglycaemia episodes by 303 new subjects and the correlations among the symptoms were subjected to a similar principal components analysis. The results of this second analysis are shown in Table 3. Using the same extraction criterion, three factors accounted for $44.9 \%$ of the total test variance. After orthogonal rotation three factors were obtained as shown in Table 3. A similar pattern emerged to that found in Study 1. All three factors explained very similar amounts of shared variance and had the same patterns of key symptom loadings as did the respective factors in Study 1. Headache did not load on the neuroglycopenic factor and palpitation and drowsiness did not load on the malaise factor, making the clarity of the neuroglycopenic, autonomic and malaise factors even greater in this attempted replication. The neuroglycopenic factor had no significant loadings from autonomic or malaise factor items. The autonomic factor had a small, but probably significant loading for incoordination, though this was much smaller than any of the loadings from the four main autonomic items. The only significant loading on the malaise factor for symptoms other than headache and nausea was a small negative loading for sweating.

Therefore, the three factor structure of the 11 hypoglycaemia items was clearly replicated in a second, independent study. The similarity of the three factors across the two studies was quantified using coefficients of congruence. Table 3 shows that these coefficients were very high for the neuroglycopenic and autonomic factors, 0.97 and 0.96 , respectively, and high for the malaise factor, at 0.88 . This provides additional evidence for the replicability of the three factor structure of hypoglycaemia symptoms across two large independent patient samples.

\section{Confirmatory factor analysis}

Conventional exploratory factor analyses indicated a three factor structure for the 11 symptoms of hypoglycaemia considered here, and cross-sample validity has been established for this structure using a second large, independent cohort of patients. The data were then submitted to structural equation modelling in which the EQS Structural Equations Program [23] was used to conduct confirmatory factor analyses separately on both groups. Thereafter, multisample confirmatory factor analysis was used to test the similarity of the covariance matrix across the two groups.

Goodness-of-fit of the three factor model. The first hypothesis to be tested was how well the three factor model, proposed on the basis of a reconsideration of previous research, fitted the two data sets in this report. To test this model, it was assumed that only three latent variables, neuroglycopenic, autonomic and malaise, accounted for the symptom covariance. Remaining variance, it was assumed, was accounted for by symptom-specific and error variance, referred to as residual variance for each symptom. Equations were written for each symptom, describing its variance in terms of contributions from one latent variable and residual variance. The series of equations describing each symptom and the latent factors constituted a 'model' to explain the symptom covariance matrix that may be tested for its goodness-of-fit to the two data sets. The models were tested using the method of Generalised Least Squares.

The equations which constituted the three factor model imposed the following constraints on symptom covariance. Confusion, drowsiness, odd behaviour, speech difficulty and incoordination were allowed to load on the neuroglycopenia factor, and their loadings on this factor were free parameters in the model. The loadings of these symptoms on the other two latent variables were fixed at zero. Sweating, palpitation, shaking and hunger were allowed to load on the autonomic factor, and their loadings on this factor were free parameters in the model. The loadings of these symptoms on the other two factors were fixed at zero. Nausea and headache were allowed to load on the third latent variable, malaise, and their loadings on the other two latent variables were fixed at zero. Symptom variance not accounted for by the above three latent variables was assumed to be captured entirely by residual variables that were specific to each symptom. These residual variables were assumed to be uncorrelated, i.e. these values were fixed at zero. The latent factors were allowed to be correlated, i. e. they were not assumed to be orthogonal. Thus, the sizes of the correlations among the latent variables were free parameters in the model. The structure of the three factor model is shown in Figure 1.

Table 4 contains a summary of the results from this analysis. The adequacy of a model's fit to the data collected may be assessed in various ways. Firstly, the average standardised residuals of both models are small and similar in size, indicating that the three factor model accounted for most of the variance in both covariance matrices. Secondly, in both cases there is a high value of $p$ for the chi-square test of goodness-of-fit. A small $p$ value, such as one of less than 0.05 , would indicate that the model had a poor fit to the data; in this case the three factor model fits both data sets well, since neither $p$ value approaches a significant value. Thirdly, the fit indices used all indicate that the model fits both data sets well. Values of 0.9 are traditionally taken to indicate an adequate model fit. For both data sets the fit indices are much greater than 0.9 , and in the case of Study 2 the Bentler-Bonett non-normed fit index, which takes account of the degrees of freedom in the model, exceeds the maximum fit value of 1 , a common occurrence with this statistic in the presence of well-fitting models.

Therefore, a model which posits neuroglycopenic, autonomic and malaise factors as latent variables underlying the covariance pattern of the 11 symptoms of hypoglycaemia has proved successful in two moderately large 


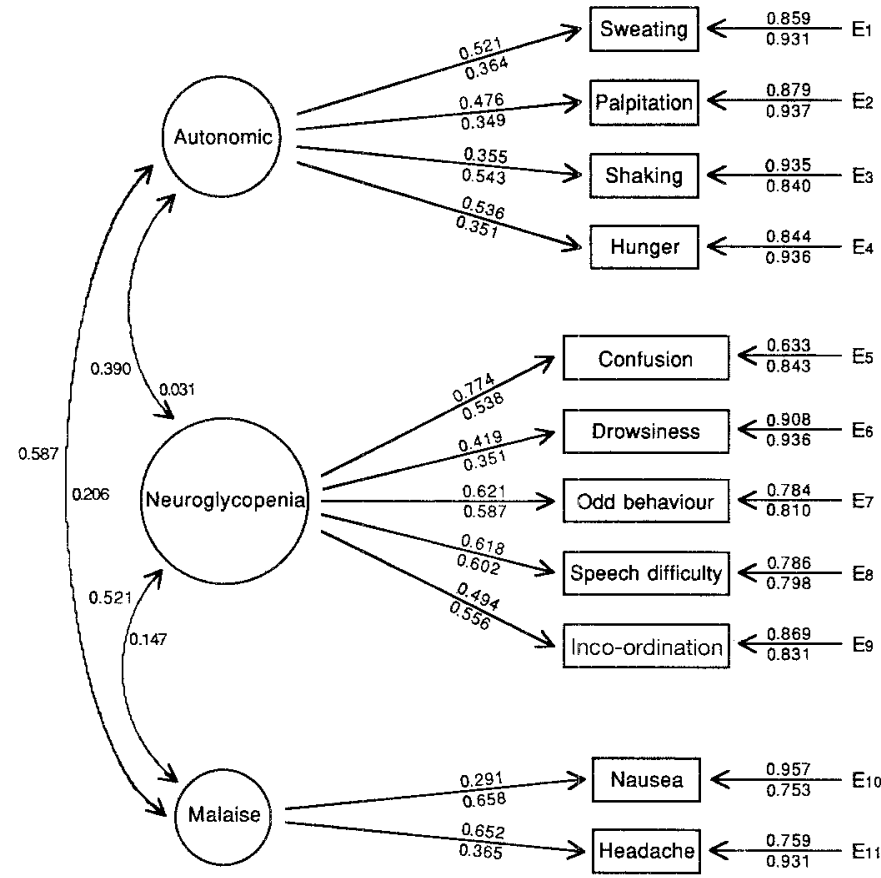

Fig. 1. Diagrammatic representation of the series of equations constructed to specify the three factor model of hypoglycaemia symptoms. As is conventional, rectangular boxes represent measured variables, circles represent latent variables, and variables marked ' $E_{n}$ ' represent the residual variance specific to the respective symptom. Straight lines represent causal pathways, with arrowheads indicating the direction of causation. Curved lines represent variables permitted to intercorrelate. Numbers adjacent to arrows represent parameter estimates for optimal model fitting, as estimated by the EQS program. Numbers above the arrows represent parameters estimated from the data in Study 1 and numbers below the arrows refer to Study 2. Note that the numbers adjacent to curved lines connecting latent variables are correlations

samples. However, this does not imply that other models might not fit the covariance matrices equally well, or better. Therefore, it is necessary to test the three factor model competitively against other models. In the EQS structural equations program the Wald and Lagrange Multiplier tests together provide an economical method of performing such tests.

Wald test. The Wald test indicates the presence of any free parameters in the model which could have been fixed at zero without a significant decrease in model fit, i.e. it gives information about the most parsimonious model that achieves maximal fit indices (Table 4). In Study 1 the Wald test indicated that none of the free parameters should be dropped, i.e. all free parameters made a significant contribution to the fit of the model. In Study 2 four free parameters could have been excluded without a decrease in model fit. The three clearest candidates for exclusion were the associations between the three latent variables. The parameters expressing their intercorrelation were significant in Study 1, indicating the presence of a 'general hypoglycaemia' factor, but this was not replicated in Study 2. The only other parameter that appeared unnecessary was the loading of headache on factor 3 in Study 2, though there was a trend for the inclusion of this parameter to improve the fit of the model. In summary, for both studies, all of the parameters expressing the loadings of symptoms on latent variables contributed significantly to the model's goodness-of-fit. The only apparent difference between the model as applied to the two studies was the intercorrelation among the latent variables, which was not a primary concern of the exercise.

Lagrange Multiplier test. The Lagrange Multiplier test indicates any parameters with fixed values that might have been freed in order to improve the fit of the model. In the case of the three factor model tested here, all fixed parameters were those fixed at zero. Therefore the Lagrange Multiplier test indicated any parameters that were assumed to be zero but might have been freed in order to allow a superior model to emerge (Table 4). In Study 1 the test found that the parameters expressing the loadings of palpitation on the malaise factor and headache on the autonomic factor might have been freed to give a significantly superior model fit. In Study 2 the test indicated that the parameter expressing the loading of incoordination on the autonomic factor should have been set at a value greater than zero.

In summary, the assumptions that symptoms had loadings exclusive to a single latent factor were largely correct except for a few small exceptions which were not consistent across the two studies. Figure 1 contains a path diagram for three factor model, with the numbers adjacent to the arrows representing the optimal parameter estimates from Studies 1 and 2. A formal test of the similarity of these parameters will be the next stage of analysis.

Multi-sample confirmatory factor analysis. The confirmatory factor analysis has added considerably to the information gathered from the exploratory factor analysis; it has suggested that the three factor model hypothesised to fit the 11 symptoms of hypoglycaemia is essentially the best possible way to construe the data. In the final analysis

Table 4. EQS results for the three factor model of hypoglycaemic symptoms from Study $1(n=295)$ and Study $2(n=303)$. Terms are explained in the Confirmatory Factor Analysis section of the text and in Table 2

\begin{tabular}{|c|c|c|}
\hline & Study 1 & Study 2 \\
\hline $\begin{array}{l}\text { Average standardised } \\
\text { residuals }\end{array}$ & 0.038 & 0.033 \\
\hline Chi-square $(d f)$ & $40.4(41)$ & $33.7(41)$ \\
\hline$p$ value of chi-square & 0.496 & 0.783 \\
\hline $\begin{array}{l}\text { Bentler-Bonett normed } \\
\text { fit index }\end{array}$ & 0.986 & 0.986 \\
\hline $\begin{array}{l}\text { Bentler Bonett non- } \\
\text { normed fit index }\end{array}$ & 1.000 & 1.004 \\
\hline Comparative fit index & 1.000 & 1.000 \\
\hline $\begin{array}{l}\text { Free parameters not } \\
\text { enhancing model fit } \\
\text { (Wald test) }\end{array}$ & None & $\begin{array}{l}\text { Factor } 1 \text { vs factor } 2 \\
\text { Factor } 1 \text { vs factor } 3 \\
\text { Factor } 2 \text { vs factor } 3 \\
\text { Headache on factor } 3\end{array}$ \\
\hline $\begin{array}{l}\text { Fixed parameters that } \\
\text { may be freed to enhance } \\
\text { model fit (Lagrange } \\
\text { Multiplier test) }\end{array}$ & $\begin{array}{l}\text { Palpitation } \\
\text { on factor } 3 \\
\text { Headache on } \\
\text { factor } 1\end{array}$ & $\begin{array}{l}\text { Incoordination on } \\
\text { factor } 1\end{array}$ \\
\hline
\end{tabular}


of this report a further hypothesis was tested. Thus far, it appeared that the same model may be said to fit both data sets tolerably well, but the similarity of the covariance matrices for the two studies may be tested for ever closer equality by making the assumption that the sizes of the respective symptom loadings on the latent factors were identical in the two data sets. Beyond the assumption that the same three factors describe the data in the two studies equally well, this procedure tests the more rigorous hypothesis that the symptoms have the same relative importance within each factor across the two studies. A further assumption included in this model was that the variances of the residual variables for all symptoms in the two data sets were equal. This adds a further degree of rigour to the test, which may be passed only if the two covariance matrices were very similar.

The method of Generalised Least Squares was used to test the above model, i.e. the assumption that the same three factor model could be used to understand the relationships among the 11 symptoms in both studies, and the constraining assumptions that: the respective symptomlatent variable loadings in the two studies were equal, and that the variances of the respective residual variables were equal in the two samples. The chi square for this model was $119.1(104 d f)$, with a $p$ value of 0.148 , indicating that the model was acceptable. The fit indices were as follows: Bentler-Bonett normed fit index $=0.978$; Bentler-Bonett non-normed fit index $=0.997$; and the comparative fit index $=0.997$. These indicate a very good fit of the model to the data. Since all possible constraints were imposed on the model, the Wald test was not appropriate. The Lagrange test was used to indicate which of the 22 imposed constraints on the model might be released to improve its fit. Five constraints might have been omitted with an associated improvement in the fit of the model. Of these, four were assumptions concerning the equality of the variances of the residual variables (palpitation, confusion, odd behaviour and nausea) across the two groups. Only one constraint concerning the equality of latent variable loadings across the two studies proved unnecessary for optimal model fit, that of confusion on the neuroglycopenia factor.

In summary, a rigorous test of the hypothesis of the similarity of the symptom loadings and residual variance equality across the two studies indicated that the findings in Study 1 were largely replicated in Study 2, and that the three factor model has been validated in this large scale cross-sample confirmatory factor analysis procedure.

\section{Discussion}

In-depth understanding of the physiological basis of symptom generation is extremely important for the comprehension of altered awareness of hypoglycaemia, a common phenomenon among long-term diabetic patients. In patients with total absence of hypoglycaemia awareness the causal mechanism is thought to be the delayed activation of the autonomic centres in the brain, such that the patient's perception of the sensory feedback from autonomic manifestations is obtunded by the significant neuroglycopenia which has by that stage supervened. In fact, most diabetic patients with altered awareness of hypoglycaemia retain some warning of its onset, with neuroglycopenic symptoms predominating. Accurate delineation of the aetiological groups of symptoms is therefore essential to allow further understanding of the alterations in awareness of hypoglycaemia.

Much of the controversy relating to the question of alteration of awareness of hypoglycaemia following transfer from porcine to human insulin centres on the claim that human insulin causes a qualitative change in hypoglycaemic symptoms from autonomic to neuroglycopenic. However, the classification of symptoms is inconsistent between studies and the selection of symptoms is more or less haphazard. This research offers a validated classification of hypoglycaemia symptoms which might be usefully adopted in further studies of hypoglycaemia symptomatology. If generally used it would facilitate the comparison of results among different centres.

In the present study 11 key symptoms were chosen from past research as being good examples of the underlying factors they represented. It was considered to be more important to provide clear factor markers than to attempt to classify every symptom that might be recorded by a patient when recalling a hypoglycaemic episode. Some symptoms have very low endorsement rates, and some symptoms amount to little more than synonyms for other symptoms; symptom selection in this report attempted to avoid these problems. Exploratory factor analysis suggested that the 11 symptoms segregated into three factors - neuroglycopenia, autonomic and malaise - and that this structure was replicated in a second large group of insulindependent patients attending the out-patient clinic. Coefficients of congruence across groups endorsed the similarity of the factors across the two groups.

Further novel and rigorous tests of the three factor model of hypoglycaemic symptoms were undertaken. Using confirmatory factor analytic methods, the three factor model was found to have highly acceptable goodnessof-fit statistics in the two groups of patients. The slight improvements that might have been made to increase the fit of the models were not replicated across the two groups, and merely reflected some low but significant factor loadings in the exploratory analyses. Formal similarity of the structure of the covariance matrices across the two groups was tested by multi-sample confirmatory factor analysis. This procedure confirmed that the respective symptom loadings across the two groups might be considered to be equal, and that the variance of most of the residual variables was equal across the two groups. Very few relaxations of this highly constrained model were required for optimal model statistics.

The important and paradoxical part played by subject numbers in these model-fitting procedures should be explained. In most statistical procedures it is the case that an increase in the numbers of subjects will result in a greater chance of accepting an experimental hypothesis, i.e. all else being equal, the experiment will have more power. Precisely the reverse is the case in structural equation modelling. When numbers of subjects are small, differences between the model and the data covariance matrix are unlikely to deliver a significant chi-square result, and a relatively poor-fitting model may be accepted. However, fit indices are likely to be low and residuals high, which should pre- 
vent hasty acceptance of a poor model. When subject numbers are large, power becomes high, and even quite small deviations of the model from the structure of the covariance matrix may produce a significant chi-square value causing, potentially, a good model to be rejected. However, the experimenter is likely to note that fit indices are very high and residuals low. Therefore, it is important to examine all aspects of the modelling procedure's results and to consider the number of subjects involved when deciding whether to accept a model. In the present study no particular problems were encountered; the patient numbers were quite large, the model was checked in an independent sample, chi-square values were non-significant, fit indices were high, and residuals were low. Therefore, the model proved satisfactory and robust.

The statistical separation of symptoms into factors was clear in this study, but the statistical approach to the allocation must be validated by other types of research. This is similar to the state of affairs in the fields of human ability and temperament, where dimensions of ability and personality are partitioned using factor analysis and then validated by examining the real life correlates of such dimensions, and the brain processes which underlie them. Also, in the field of hypoglycaemia, it will be important to discover the clinical associations of symptom groupings; it will be of interest to note how the factors alter, if at all, with age, duration of diabetes, and with alterations in the type or regimen of insulin treatment, or both. Additionally, the statistical approach must be validated by uncovering more clearly the physiological mechanisms underlying symptom generation.

In the meantime we encourage standardisation across research centres by use of the 11 symptoms which constitute the validated three factor model. In order to encourage improvement in the model the 11 symptoms may be identified as the Edinburgh Hypoglycaemia Scale. A single response format to this 11 -item scale is not suggested, because different types of study will require different formats. A binary response format, as was used in the present studies, might be sufficient for retrospective research. Alternatively, a seven point scale or a visual analogue scale, with anchors such as 'Not at all' and 'Very severely' might be used to index patients' experiences of the symptoms in the acute situation.

\section{References}

1. Fletcher AA, Campbell WR (1922) The blood sugar following insulin administration and the symptom complex-hypoglycemia. J Metab Res 2: 637-649

2. Goldgewicht C, Slama G, Papoz L, Tchobroutsky G (1983) Hypoglycaemic reactions in 172 type 1 (insulin-dependent) diabetic patients. Diabetologia 24: 95-99

3. Mutch WJ, Dingwall-Fordyce I (1985) Is it a hypo? Knowledge of the symptoms of hypoglycaemia in elderly diabetic patients. Diabetic Med 2: 54-56

4. Aman J, Karlsson I, Wranne L (1989) Symptomatic hypoglycaemia in childhood diabetes: a population-based questionnaire study. Diabetic Med 6: 257-261

5. Hepburn DA, Eadington DW, Patrick AW, Colledge NR, Frier BM (1989) Symptomatic awareness of hypoglycaemia: does it change on transfer from animal to human insulin? Diabetic Med 6: $586-590$
6. Ward CM, Stewart AW, Cutfield RG (1990) Hypoglycaemia in insulin-dependent diabetic patients attending an outpatients' clinic. N Z Med J 103: 339-341

7. Mühlhauser I, Heinemann L, Fritsche E, Von Lennep K, Berger M (1991) Hypoglycemic symptoms and frequency of severe hypoglycaemia in patients treated with human and animal insulin preparations. Diabetes Care 14: 745-749

8. Hepburn DA, Deary IJ, Frier BM (1992) Classifications of symptoms of hypoglycaemia in insulin-treated diabetic patients using factor analysis: relationship to hypoglycaemia unawareness. Diabetic Med 9: 70-75

9. Macfarlane PI, Walters M, Stutchfield P, Smith CS (1989) A prospective study of symptomatic hypoglycaemia in childhood diabetes. Diabetic Med 6: 627-630

10. Pramming S, Thorsteinsson B, Bendtson I, Binder C (1991) Symptomatic hypoglycaemia in 411 type 1 diabetic patients. Diabetic Med 8:217--222

11. Pennebaker JW, Cox DJ, Gonder-Frederick L, Wunsch MG, Evans WS, Pohl S (1981) Physical symptoms related to blood glucose in insulin-dependent diabetics. Psychosom Med 43: 489-500

12. Cox DJ, Gonder-Frederick L, Pohl S, Pennebaker JW (1983) Reliability of symptom-blood glucose relationships among insulindependent adult diabetics. Psychosom Med 45:357-360

13. Cox, DJ, Clarke, WL, Gonder-Frederick Let al. (1985) Accuracy of perceiving blood glucose in IDDM. Diabetes Care 8: 529-536

14. Corrall RJM, Frier BM, Davidson NMcD, Hopkins WM, French EB (1983) Cholinergic manifestations of the acute autonomic reaction to hypoglycaemia in man. Clin Sci 64: 49-53

15. Holmes CS, Hayford JT, Gonzalez JL, Weydert JA (1983) A survey of cognitive functioning at different glucose levels in diabetic persons. Diabetes Care 6: 180-185

16. Fellows IW, MacDonald IA, Wharrad HJ, Birmingham AT (1986) Low plasma concentrations of adrenaline and physiological tremor in man. J Neurol Neurosurg Psych 49: 396-399

17. Pramming S, Thorsteinsson B, Theilgaard A, Pinner EM, Binder C (1986) Cognitive function during hypoglycaemia in type 1 diabetes mellitus. BMJ 292: 647-650

18. Berger WG, Keller U, Honegger B, Jaeggi E (1989) Warning symptoms of hypoglycaemia during treatment with human and porcine insulin in diabetes mellitus. Lancet I: 1041-1044

19. Heine RJ, Van der Heyden EAP, Van der Veen EA (1989) Responses to human and porcine insulin in healthy subjects. Lancet II: $946-949$

20. Frier BM, Hepburn DA (1989) Plasma noradrenaline, human insulin, and hypoglycaemia. Lancet II: 1269 (Letter)

21. Hepburn DA, Frier BM (1989) Hypoglycaemia unawareness and human insulin. Lancet I: 1394 (Letter)

22. Hepburn DA, Deary IJ, Frier BM, Patrick AW, Quinn JD, Fisher BM (1991) Symptoms of acute insulin-induced hypoglycemia in humans with and without IDDM. A factor analysis approach. Diabetes Care 14: 949-957

23. Bentler PM (1989) EQS structural equations program manual. BMDP Statistical Software, Los Angeles

24. Hepburn DA, Patrick AW, Eadington DW, Ewing DJ, Frier BM (1990) Unawareness of hypoglycaemia in insulin-treated diabetic patients: prevalence and relationship to autonomic neuropathy. Diabetic Med 7:711-717

25. Child D (1990) The essentials of factor analysis, 2 nd edn. Cassell, London

Received: 22 December 1992

and in revised form: 29 March 1993

Dr. I.J.Deary

Department of Psychology

University of Edinburgh

7, George Square

Edinburgh EH8 9JZ

Scotland, UK 